University of Wollongong

Research Online

Australian Institute for Innovative Materials -

Papers

Australian Institute for Innovative Materials

$1-1-2017$

Anisotropic thermomagnetic avalanche activity in field-cooled superconducting films

F Colauto

Federal University of Sao Carlos

D Carmo

Federal University of Sao Carlos

A M H De Andrade

Federal University of Rio Grande do Sul

A A M Oliveira

Federal University of Sao Carlos

Wilson Aires Ortiz

Federal University of Sao Carlos

See next page for additional authors

Follow this and additional works at: https://ro.uow.edu.au/aiimpapers

Part of the Engineering Commons, and the Physical Sciences and Mathematics Commons

Research Online is the open access institutional repository for the University of Wollongong. For further information contact the UOW Library: research-pubs@uow.edu.au 


\title{
Anisotropic thermomagnetic avalanche activity in field-cooled superconducting films
}

\author{
Abstract \\ The electrodynamic behavior of isotropic superconducting $\mathrm{Nb}$ films cooled below their critical \\ temperature in the presence of in-plane applied magnetic fields is investigated using magneto-optical \\ imaging. A specially designed local flux injector is used to show that the frozen-in in-plane vortices \\ strongly guide and enhance the penetration of perpendicular vortices, whereas their penetration across \\ the array of in-plane vortices is essentially unchanged. This result provides the key to understanding why \\ field-cooled square superconducting films show anisotropic nucleation of flux avalanches (jumps) along \\ the four edges. The explanation is based on an analytical model for thermomagnetic avalanche \\ nucleation in type-II superconducting films, and allows one to understand the entire scenario of different \\ flux dynamics observed experimentally. \\ Disciplines \\ Engineering | Physical Sciences and Mathematics

\section{Publication Details} \\ Colauto, F., Carmo, D., De Andrade, A. M. H., Oliveira, A. A. M., Ortiz, W. A. \& Johansen, T. H. (2017). \\ Anisotropic thermomagnetic avalanche activity in field-cooled superconducting films. Physical Review B: \\ Condensed Matter and Materials Physics, 96 (6), 060506-1-060506-5.

\section{Authors} \\ F Colauto, D Carmo, A M H De Andrade, A A M Oliveira, Wilson Aires Ortiz, and Tom H. Johansen
}




\title{
Anisotropic thermomagnetic avalanche activity in field-cooled superconducting films
}

\author{
F. Colauto, ${ }^{1}$ D. Carmo, ${ }^{1}$ A. M. H. de Andrade, ${ }^{2}$ A. A. M. Oliveira, ${ }^{3}$ W. A. Ortiz, ${ }^{1}$ and T. H. Johansen ${ }^{4,5}$ \\ ${ }^{1}$ Departamento de Física, Universidade Federal de São Carlos, 13565-905, São Carlos, SP, Brazil \\ ${ }^{2}$ Instituto de Física, Universidade Federal do Rio Grande do Sul, 91501-970, Porto Alegre, RS, Brazil \\ ${ }^{3}$ Instituto Federal de Educação, Ciência e Tecnologia de São Paulo, 13565-905, São Carlos, SP, Brazil \\ ${ }^{4}$ Department of Physics, University of Oslo, P.O. Box 1048 Blindern, 0316 Oslo, Norway \\ ${ }^{5}$ Institute for Superconducting and Electronic Materials, University of Wollongong, Northfields Avenue, Wollongong, NSW 2522, Australia
}

(Received 11 May 2017; revised manuscript received 19 June 2017; published 16 August 2017)

\begin{abstract}
The electrodynamic behavior of isotropic superconducting $\mathrm{Nb}$ films cooled below their critical temperature in the presence of in-plane applied magnetic fields is investigated using magneto-optical imaging. A specially designed local flux injector is used to show that the frozen-in in-plane vortices strongly guide and enhance the penetration of perpendicular vortices, whereas their penetration across the array of in-plane vortices is essentially unchanged. This result provides the key to understanding why field-cooled square superconducting films show anisotropic nucleation of flux avalanches (jumps) along the four edges. The explanation is based on an analytical model for thermomagnetic avalanche nucleation in type-II superconducting films, and allows one to understand the entire scenario of different flux dynamics observed experimentally.
\end{abstract}

DOI: 10.1103/PhysRevB.96.060506

The gradual penetration of magnetic flux in films of type-II superconductors experiencing an increasing external magnetic field can be interrupted by the onset of dramatic flux avalanches. These events, also called flux jumps, nucleate at points along the film edge, and from there, the flux invades the film in the form of dendritic structures with branches advancing at speeds up to $100 \mathrm{~km} / \mathrm{s}$ [1-3]. Using magneto-optical imaging (MOI) [4], this phenomenon has been observed in films of many superconducting materials, such as $\mathrm{Nb}$ [5], $\mathrm{Pb}$ [6], $\mathrm{MgB}_{2}$ [7], $\mathrm{Nb}_{3} \mathrm{Sn}$ [8], $\mathrm{YNi}_{2} \mathrm{~B}_{2} \mathrm{C}$ [9], $\mathrm{NbN}$ [10], MoGe [11], and MoSi [12].

Films of $\mathrm{YBa}_{2} \mathrm{Cu}_{3} \mathrm{O}_{x}$, however, show remarkable stability. For a long time the only known way to trigger avalanche events in this material was by applying a laser pulse to perturb the flux-filled film locally [1]. More recently, Baziljevich et al. $[13,14]$ showed that also $\mathrm{YBa}_{2} \mathrm{Cu}_{3} \mathrm{O}_{x}$ films become unstable in magnetic fields when applied at rates near $3000 \mathrm{~T} / \mathrm{s}$. They also found that during these avalanches, the $\mathrm{YBa}_{2} \mathrm{Cu}_{3} \mathrm{O}_{x}$ decomposed and evaporated, leaving ditches in the film as permanent traces of the advancing avalanche front-a unique evidence for the thermomagnetic nature of the instability [15-18].

Another remarkable observation was reported by Albrecht et al. [19], where a square $\mathrm{MgB}_{2}$ film was deposited on a vicinal substrate, creating $6 \%$ anisotropy in the critical current density. Although small, this anisotropy resulted in dramatic changes in the avalanche activity as a function of temperature $T$. At $T$ well below $10 \mathrm{~K}$, the avalanches nucleated evenly from all four edges of the film, and when approaching $10 \mathrm{~K}$, they occurred only from the pair of edges where the larger critical current density was flowing. Then, above $10 \mathrm{~K}$, avalanches never appeared. This peculiar behavior was fully explained using theoretical results obtained in works by Denisov et al. $[17,18]$.

Quite recently, Vlasko-Vlasov et al. [20] reported MOI investigations of a square $\mathrm{Nb}$ film initially cooled below $T_{c}$ in the presence of an in-plane magnetic field $H_{\|}$. When then a perpendicular field $H_{\perp}$ was applied, it was found at $6 \mathrm{~K}$ that the flux penetration was gradual, and strongly anisotropic. At $5 \mathrm{~K}$, the penetration took the form of dendritic avalanches, also displaying distinct anisotropic features, namely, with $H_{\|}$applied along any pair of parallel edges, the avalanches nucleated only from the edges aligned with the field. Those edges were also seen to have the steepest gradient in the density of penetrated perpendicular flux. A qualitative explanation for the intriguing observation was given by drawing analogy to avalanches occurring along slopes in granular media.

In the present Rapid Communication, the anisotropic avalanche behavior reported in Ref. [20] is fully explained from equations governing the electrodynamics of type-II superconducting films. The explanation is based also on different MOI observations where we make active use of local flux injections [21] into $\mathrm{Nb}$ films with $H_{\|}$-induced anisotropy. These results allow us to adopt an analytical approach related to that used in Ref. [19], thus generalizing the applicability of the stability analysis of the coupled nonlinear and nonlocal equations governing the thermomagnetic instability in film superconductors.

The first question to address is whether the anisotropic regular flux penetration in $\mathrm{Nb}$ films with frozen-in $H_{\|}$is due to reduced pinning of the perpendicular vortices moving parallel to the in-plane ones. Or is it due to enhanced pinning of the incoming vortices as they move across the array of in-plane vortices? In principle, both effects could also be active simultaneously.

To resolve the ambiguity we made use of the flux injector design shown in Fig. 1. Samples of this shape were prepared from 200-nm-thick $\mathrm{Nb}$ films grown on $\mathrm{Si}(100)$ substrates by magnetron sputtering in a UHV system with a base pressure of less than $2 \times 10^{-8}$ Torr. The films have a critical temperature of $T_{c}=9.1 \mathrm{~K}$, and patterning was performed using optical lithography. By passing an applied current $i_{\text {app }}$ in the legs extending from the lower edge, a controllable amount of magnetic flux can be injected into the square from the point where the two legs meet [21]. The sides of the square are $2.5 \mathrm{~mm}$ long. 

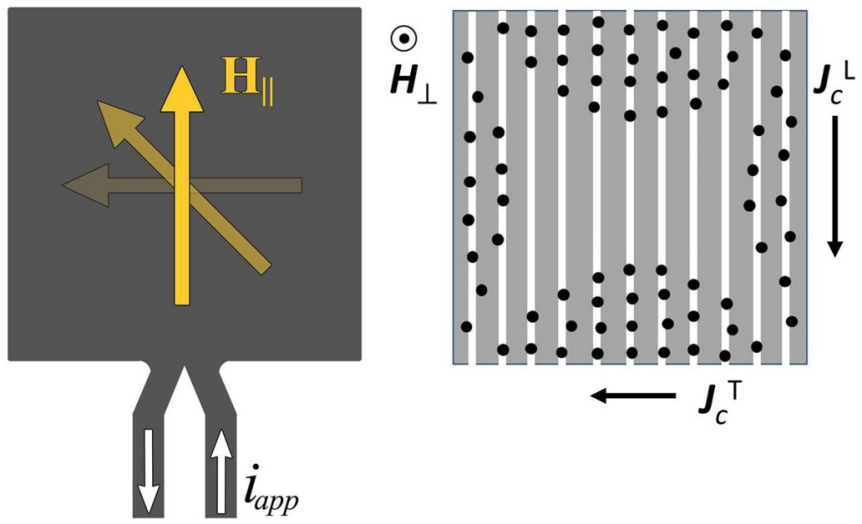

FIG. 1. Left: Outline of the $\mathrm{Nb}$ film sample with a flux injector at the lower edge. Indicated by arrows are the orientations of in-plane magnetic fields applied in this work. Right: Illustration of anisotropic penetration of perpendicular vortices (black dots) in the presence of frozen-in in-plane vortices (white lines) [20].

To observe the flux penetration into the sample, we used a magneto-optical imaging (MOI) setup where a thin plate of Faraday rotating Bi-substituted ferrite-garnet film with in-plane magnetization served as the sensor [22]. The sensor plate was placed directly on top of the $\mathrm{Nb}$ film, and when viewed in a polarized light microscope with crossed polarizers, the image brightness represented a direct map of the magnetic flux density distribution [23]. The objective lens used in this work was a $5 \mathrm{X}$ MPlanFL Olympus, and images were recorded using a Qimaging Retiga 4000R CCD camera. Its pixel scale was calibrated based on a Pelcotec TM critical dimension magnification standard (CDMS). The image analysis was carried out using the IMAGE J 1.48 software. A resistive coil was placed around the optical cryostat to apply perpendicular fields $H_{\perp}$. In-plane fields $H_{\|}$were generated by a pair of permanent magnets mounted on a rotatable stage, allowing $H_{\|}$to be applied in various directions (see Fig. 1, left panel). Current pulses were provided by a Keithley-2635 current source.

Presented in Fig. 2 are magneto-optical images of the sample recorded after initial cooling to $7.0 \mathrm{~K}$, a temperature where avalanche events do not occur in $\mathrm{Nb}$ films. A perpendicular field, $H_{\perp}=5 \mathrm{Oe}$, was then applied only to provide contrast for making the sample contour visible. Finally, a current pulse of magnitude $i_{\text {app }}=0.6 \mathrm{~A}$ and duration $200 \mathrm{~ms}$ was applied to the injector, creating the local flux penetration seen in the images, where the brightness is a direct measure of the perpendicular flux density.

Figure 2(a) shows the flux distribution after the flux injection took place with $H_{\|}=0$, thus serving as a reference case. From the image one sees that the current pulse caused perpendicular flux to invade a nearly semicircular region of the square sample.

Shown in Fig. 2(b) is the result of the same current pulse when the field $H_{\|}=1 \mathrm{kOe}$, directed as indicated by the arrow, was applied during the initial cooling. Evidently, the frozen-in field caused a strong enhancement of the flux penetration in the direction of $H_{\|}$. Equally important here is that the penetration depth of the flux-injected region is essentially the same as in Fig. 2(a). This shows that when the current-induced
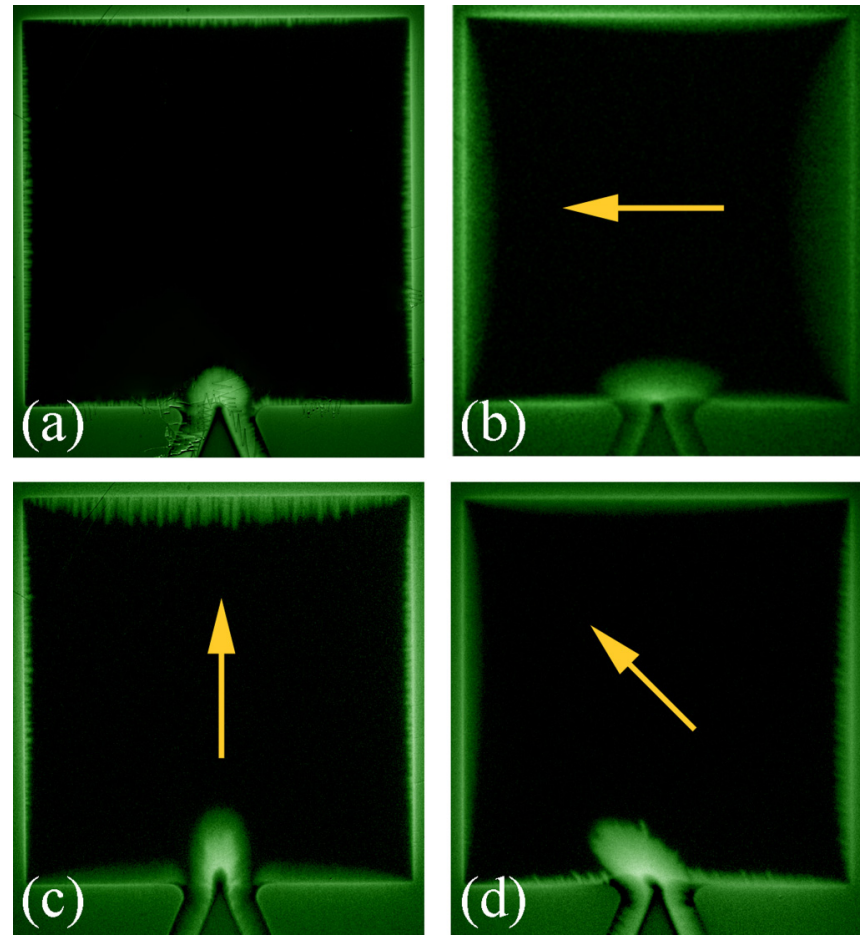

FIG. 2. Magneto-optical images of the sample cooled to $7.0 \mathrm{~K}$ before a current pulse was applied in the injector. (a) shows the flux distribution after initial zero-field cooling. In (b)-(d), an in-plane field, $H_{\|}=1 \mathrm{kOe}$. oriented in three different directions indicated by the arrows, was applied during the initial cooling.

vortices traverse the in-plane array of frozen-in ones, the injected vortices experience practically no additional pinning. Moreover, since the penetrated region is strongly elongated in the direction of $H_{\|}$, one concludes that the pinning in that direction is significantly reduced.

Figure 2(c) shows the result when the $H_{\|}$was oriented vertically in the figure. The tendency described above is repeated here, i.e., parallel to $H_{\|}$the injected flux penetrates much deeper than in Fig. 2(a), while the transverse width of the injected area is essentially the same. Finally, Fig. 2(d) displays the result with $H_{\|}$oriented along a diagonal of the square sample, and confirms that the dominant effect of $H_{\|}$is to guide the flux injection.

These observations allow us to conclude that the anisotropy in the flux penetration is largely due to reduced pinning of the vortices moving parallel or antiparallel to the frozen-in $H_{\|}$. This translates directly into an anisotropy in the critical sheet current. Let $J_{c}^{L}$ and $J_{c}^{T}$ denote the critical sheet current flowing in the directions along and transverse to the $H_{\|}$, respectively (see Fig. 1, right panel). From the injection images in Fig. 2 and qualitative use of Ampere's law, we conclude that $J_{c}^{L}$ is essentially constant, whereas $J_{c}^{T}$ becomes smaller with larger applied $H_{\|}$.

Consider next the flux penetration at a lower temperature in a similar $\mathrm{Nb}$ film shaped as a plain square with $2.5-\mathrm{mm}$ sides. The film, having the same thickness as the sample with the injector, was initially cooled to $T=2.5 \mathrm{~K}$ in the presence of in-plane magnetic fields. Then, a perpendicular field of 

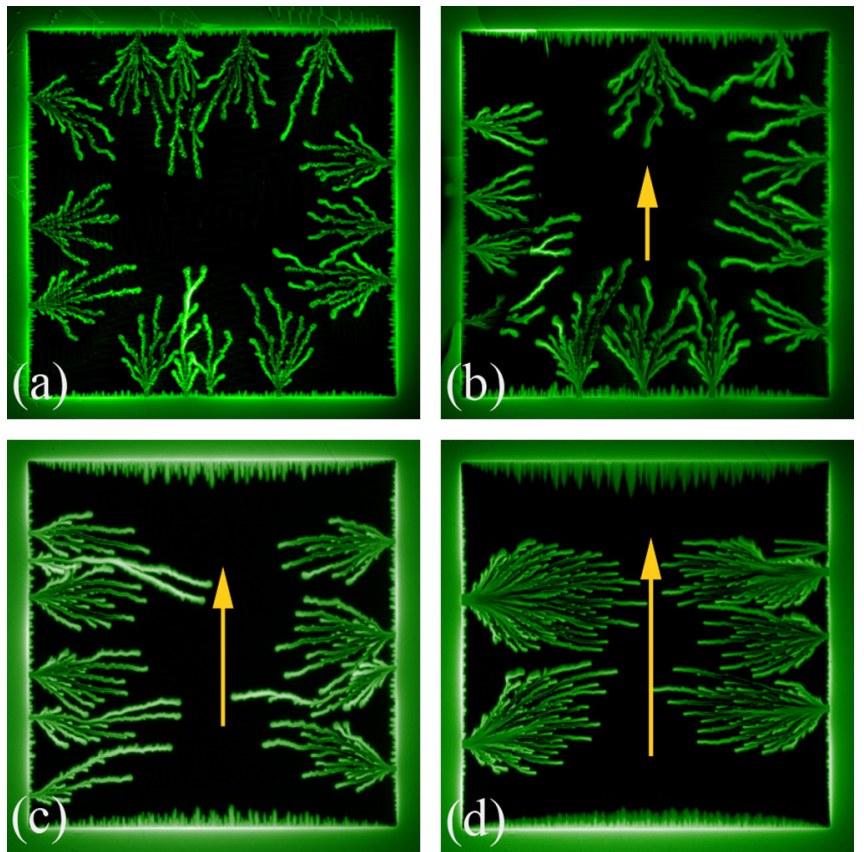

FIG. 3. Magneto-optical images of the penetration of perpendicular flux in a square $\mathrm{Nb}$ film, where in-plane fields, indicated by the arrows, were applied during the initial cooling to $2.5 \mathrm{~K}$. In (a)-(d), the $H_{\|}$was $0,0.7,1.0$, and $1.5 \mathrm{kOe}$, respectively.

$H_{\perp}=38$ Oe was applied, creating the flux distribution seen in the images presented in Fig. 3.

Figure 3(a) shows the flux penetration into the initially zero-field-cooled sample, and one sees that the penetration at this temperature is dominated by dendritic avalanches. Each branched structure is the result of one avalanche event, which started from the point at the edge where the tree structure is rooted. In this panel one sees that the avalanches nucleated from locations quite equally distributed between all the four edges.

In Fig. 3(b), the isotropic symmetry is broken as the majority of avalanches here nucleate from the pair of edges oriented vertically in the figure. Then, in Fig. 3(c), the anisotropy is complete, as no avalanches nucleate along the horizontal edges. When increasing the $H_{\|}$further [see Fig. 3(d)], the full anisotropy remains.

To explain this striking behavior we make use of results from theoretical analyses of the thermomagnetic instability in superconducting films thermally coupled to their substrate $[17,18]$. The analyses consider a film strip of width $2 w$ and thickness $d \ll w$ placed in an increasing perpendicular magnetic field, creating a critical state in the flux-penetrated region near the edges. By solving the Maxwell and thermal diffusion equations, it was shown that for small perpendicular fields there is no solution for perturbations growing with time, implying a stable situation. As the field increases, the flux distribution can become unstable, with the fastest growing perturbation having a nonzero wave vector along the film edge. This implies an instability developing in the form of a narrow finger perpendicular to the edge, a scenario corresponding to the early stage of the observed flux dendrites.

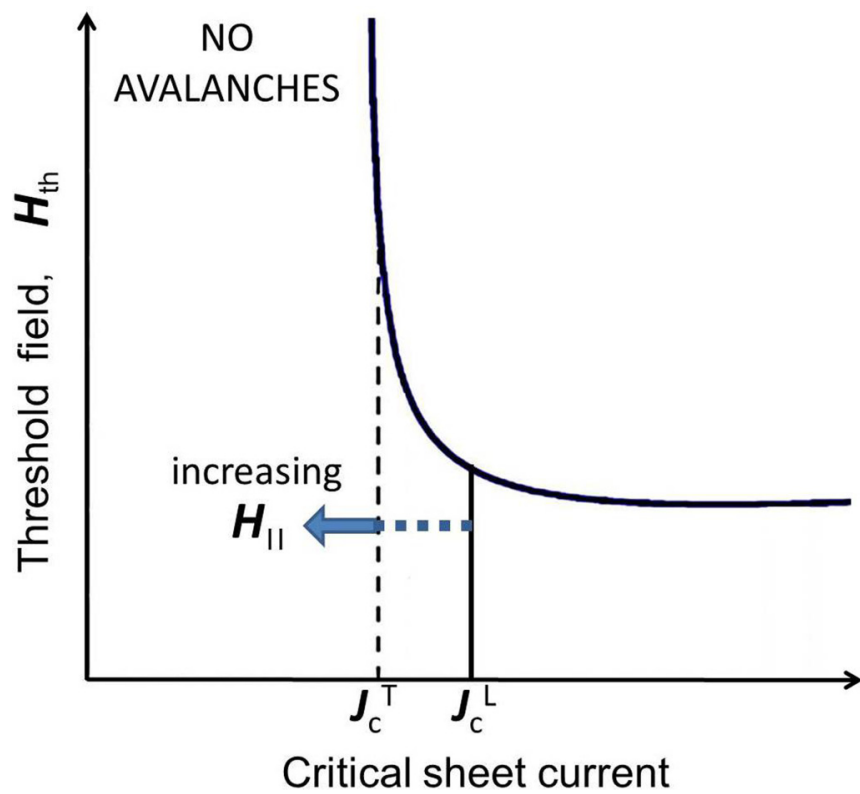

FIG. 4. Generic curve for threshold applied perpendicular field for the onset of thermomagnetic avalanche activity in superconducting films vs their critical sheet current.

Within this model, a film of thickness $d$ becomes unstable when the flux penetration depth $\ell$ reaches the value given by

$$
\ell=\frac{\pi}{2} \sqrt{\frac{\kappa T^{*} d}{J_{c} E}}\left(1-\sqrt{\frac{2 h_{0} T^{*}}{n J_{c} E}}\right)^{-1} .
$$

Here, $T^{*} \equiv-\left(\partial \ln J_{c} / \partial T\right)^{-1}, \kappa$ is the thermal conductivity, and $h_{0}$ is the coefficient of heat transfer between the film and the substrate. The parameter $n \gg 1$ characterizes the nonlinear current-voltage curve of the superconductor, described by the commonly used relation for the electrical field, $E \propto J^{n}$.

The threshold value for the applied perpendicular field $H_{\text {th }}$ can then be found by combining Eq. (1) with the Bean model expression for the flux penetration depth in a thin strip of half-width $w$ [24,25], which gives

$$
H_{\mathrm{th}}=\frac{J_{c}}{\pi} \operatorname{arccosh}\left(\frac{1}{1-\ell / w}\right) .
$$

Plotted in Fig. 4 as a solid curve is the generic relation between the threshold field and $J_{c}$, based on the two equations above. Included in the figure are also two line segments representing the two critical sheet currents $J_{c}^{L}$ and $J_{c}^{T}$. The lines are drawn vertically, to stay within the Bean model approximation.

According to the present MOI observations, the solid line, representing $J_{c}^{L}$, should remain fixed in the graph, being essentially independent of the magnitude of $H_{\|}$. The dashed line, representing $J_{c}^{T}$, should, for the isotropic case $H_{\|}=0$, obviously overlap with $J_{c}^{L}$. Then, for increasing $H_{\|}$, the $J_{c}^{T}$ gradually decreases [26], and the dashed line shifts to the left in the graph. The threshold field will then increase for avalanche nucleation along the two edges where $J_{c}^{T}$ flows. At the same time, the threshold field at the other two edges remains unchanged. Thus, more avalanche events are expected to start there, in full accord with the anisotropy seen in Fig. 3(b). 


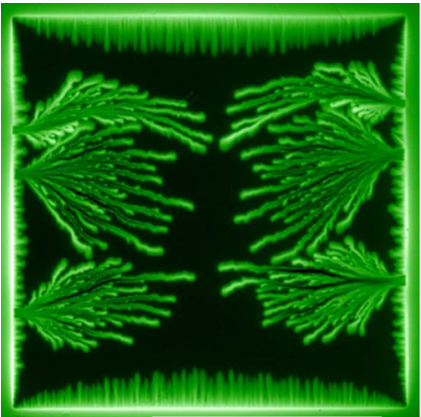

in-plane vortices ||||||||||||||||||||

FIG. 5. Magneto-optical images illustrating similarities in the flux avalanches occurring in a $\mathrm{Nb}$ film with frozen-in in-plane vortices (left), and a $\mathrm{MgB}_{2}$ film grown on a vicinal substrate (right) [19]. Both images were taken at the respective maximum temperature for avalanches to occur.

Then, as $H_{\|}$increases further, the dashed line in Fig. 4 will at some point enter the region where avalanches can no longer occur. Thus, avalanches will then only nucleate from the two edges along which the $J_{c}^{L}$ flows, again in full agreement with the MOI observations. We therefore conclude that the entire scenario of different avalanche activities in a square superconducting film with various degrees of field-induced anisotropy has been explained based on an adequate theoretical model.

It is interesting to compare the morphologies of the avalanches observed in the present experiments with those in the work on $\mathrm{MgB}_{2}$ [19]. Figure 5 presents in the left panel a magneto-optical image of our $\mathrm{Nb}$ film at $T=4.5 \mathrm{~K}$, which is close to the highest temperature where the penetration is dendritic in this film. During the cooling, the field $H_{\|}=$ $1.0 \mathrm{kOe}$ was frozen in, as indicated in the schematic shown below the images. Then, the field $H_{\perp}=45$ Oe was applied, triggering the large and strongly branching avalanches from the pair of edges that are parallel to the frozen-in flux.

The right panel shows an image of the $\mathrm{MgB}_{2}$ film at $T=$ $10 \mathrm{~K}$, which is the border line temperature between dendritic and regular flux penetration. Here, the applied field was $H_{\perp}=160 \mathrm{Oe}$, and the terrace structure of the vicinal substrate is indicated below the image. Although the $\mathrm{MgB}_{2}$ film shows a significant edge roughness, and a higher concentration of pointlike defects, all the main characteristics in the two images are essentially the same, and despite the fact that the physical origins of the anisotropy are completely different.
This suggests a remarkable universality in the theoretical model applied here for the onset conditions of thermomagnetic avalanches in superconducting films.

Finally, note that in a recent work [27] the formulas above, represented in the graph of Fig. 4, were generalized by including in the linear stability analysis also modes with complex instability increments, i.e., scenarios of oscillatory precursor behavior were also considered. It was found there that the threshold perpendicular field can then be expressed as

$$
H_{\mathrm{th}}=\frac{J_{c}}{\pi} \operatorname{arctanh}\left(\frac{h T^{\star}}{n w J_{c} \mu_{0} H_{\perp}^{\prime}(t)}\right),
$$

where $H_{\perp}^{\prime}(t)$ is the ramp rate of the applied field. It is readily verified that the curve representing the above relation between $H_{\text {th }}$ and $J_{c}$ has the same qualitative shape as the graph shown in Fig. 4. Thus, the present analysis, making use of the generic graph in that figure, has an even broader basis of applicability. However, it still remains to be found which types of modes are actually responsible for triggering this instability in different materials under various conditions.

In conclusion, we have investigated magnetic flux penetration in isotropic square $\mathrm{Nb}$ superconducting films cooled below $T_{c}$ in the presence of externally applied in-plane magnetic fields. Using magneto-optical imaging, it was found that the frozen-in field creates a tunable extrinsic anisotropy in the critical sheet current, leading to a corresponding anisotropy in the penetration of perpendicular flux. In square films cooled to $2.5 \mathrm{~K}$, the flux penetration was largely dominated by avalanche dynamics, and with increasing in-plane fields the avalanche activity became increasingly anisotropic, with the majority of avalanches nucleating from edges oriented parallel to the frozen-in field. This sequence of behaviors observed for a range of in-plane fields was fully explained theoretically. Two aspects of the present Rapid Communication are worth emphasizing: (i) the universality of the thermomagnetic model, as demonstrated by its ability to explain anisotropic avalanche activities in superconductors where the origin and characteristics of their anisotropy are quite different, and (ii) the efficiency of using a local flux injector, whose assistance provided a different way to unambiguously reveal flux guidance effects.

The $\mathrm{Nb}$ films were grown in Laboratório de Conformação Nanométrica (LCN-IF-UFRGS), and the lithography was made in Laboratório de Microfabricação (LMF/LNNano/CNPEM). The work was partially supported by the Sao Paulo Research Foundation (FAPESP) Grants No. 2013/16.097-3 and No. 2015/06.085-3, the Brazilian National Council for Scientific and Technological Development $(\mathrm{CNPq})$, the Brazilian program Science without Borders, as well as the CAPES-SIU-2013/10046 project "Complex fluids in confined environments". T.H.J. acknowledges the support from S. X. Dou at ISEM, University of Wollongong, during his sabbatical leave from Oslo.
[1] P. Leiderer, J. Boneberg, P. Brull, V. Bujok, and S. Herminghaus, Phys. Rev. Lett. 71, 2646 (1993).

[2] B. Biehler, B.-U. Runge, P. Leiderer, and R. G. Mints, Phys. Rev. B 72, 024532 (2005).
[3] P. Mikheenko, T. H. Johansen, S. Chaudhuri, I. J. Maasilta, and Y. M. Galperin, Phys. Rev. B 91, 060507(R) (2015).

[4] C. Jooss, J. Albrecht, H. Kuhn, S. Leonhardt, and H. Kronmller, Rep. Prog. Phys. 65, 651 (2002). 
[5] C. A. Duràn, P. L. Gammel, R. E. Miller, and D. J. Bishop, Phys. Rev. B 52, 75 (1995).

[6] V. Vlasko-Vlasov, U. Welp, V. Metlushko, and G. W. Crabtree, Physica C 341, 1281 (2000).

[7] T. H. Johansen, M. Baziljevich, D. V. Shantsev, P. E. Goa, Y. M. Galperin, W. N. Kang, H. J. Kim, E. M. Choi, M.-S. Kim, and S. I. Lee, Europhys. Lett. 59, 599 (2002).

[8] I. A. Rudnev, S. V. Antonenko, D. V. Shantsev, T. H. Johansen, and A. E. Primenko, Cryogenics 43, 663 (2003).

[9] S. C. Wimbush, B. Holzapfel, and C. Jooss, J. Appl. Phys. 96, 3589 (2004).

[10] I. A. Rudnev, D. V. Shantsev, T. H. Johansen, and A. E. Primenko, Appl. Phys. Lett. 87, 042502 (2005).

[11] M. Motta, F. Colauto, J. I. Vestgarden, J. Fritzsche, M. Timmermans, J. Cuppens, C. Attanasio, C. Cirillo, V. V. Moshchalkov, J. Van de Vondel, T. H. Johansen, W. A. Ortiz, and A. V. Silhanek, Phys. Rev. B 89, 134508 (2014).

[12] F. Colauto, M. Motta, A. Palau, M. G. Blamire, T. H. Johansen, and W. A. Ortiz, IEEE Trans. Appl. Supercond. 25, 7500704 (2015).

[13] M. Baziljevich, E. Baruch-El, T. H. Johansen, and Y. Yeshurun, Appl. Phys. Lett. 105, 012602 (2014)

[14] E. Baruch-El, M. Baziljevich, B. Y. Shapiro, T. H. Johansen, A. Shaulov, and Y. Yeshurun, Phys. Rev. B 94, 054509 (2016).

[15] A. L. Rakhmanov, D. V. Shantsev, Y. M. Galperin, and T. H. Johansen, Phys. Rev. B 70, 224502 (2004).

[16] I. S. Aranson, A. Gurevich, M. S. Welling, R. J. Wijngaarden, V. K. Vlasko-Vlasov, V. M. Vinokur, and U. Welp, Phys. Rev. Lett. 94, 037002 (2005).
[17] D. V. Denisov, A. L. Rakhmanov, D. V. Shantsev, Y. M. Galperin, and T. H. Johansen, Phys. Rev. B 73, 014512 (2006).

[18] D. V. Denisov, D. V. Shantsev, Y. M. Galperin, E.-M. Choi, H.-S. Lee, S.-I. Lee, A. V. Bobyl, P. E. Goa, A. A. F. Olsen, and T. H. Johansen, Phys. Rev. Lett. 97, 077002 (2006).

[19] J. Albrecht, A. T. Matveev, J. Strempfer, H.-U. Habermeier, D. V. Shantsev, Y. M. Galperin, and T. H. Johansen, Phys. Rev. Lett. 98, 117001 (2007).

[20] V. K. Vlasko-Vlasov, F. Colauto, A. A. Buzdin, D. Carmo, A. M. H. Andrade, A. A. M. Oliveira, W. A. Ortiz, D. Rosenmann, and W.-K. Kwok, Phys. Rev. B 94, 184502 (2016).

[21] D. Carmo, F. Colauto, A. M. H. de Andrade, A. A. M. Oliveira, W. A. Ortiz, and T. H. Johansen, Supercond. Sci. Technol. 29 095003 (2016).

[22] L. E. Helseth, R. W. Hansen, E. I. Ilyashenko, M. Baziljevich, and T. H. Johansen, Phys. Rev. B 64, 174406 (2001).

[23] V. K. Vlasko-Vlasov, U. Welp, G. W. Crabtree, and V. I. Nikitenko, NATO Sci. Ser. E 356, 205 (1999).

[24] E. H. Brandt and M. Indenbom, Phys. Rev. B 48, 12893 (1993).

[25] E. Zeldov, J. R. Clem, M. McElfresh, and M. Darwin, Phys. Rev. B 49, 9802 (1994).

[26] Separate measurements have shown that the anisotropy in the $J_{c}$ 's is well described by $J_{c}^{T}=J_{c}^{L} /\left(1+c H_{\|}^{3}\right)$, where $c=$ $8 \times 10^{-10} \mathrm{Oe}^{-3}$.

[27] J. I. Vestgården, Y. M. Galperin, and T. H. Johansen, Phys. Rev. B 93, 174511 (2016). 